\title{
Interhospital Transfer Handoff Practices Among US Tertiary Care Centers: A Descriptive Survey
}

\author{
Dana J. Herrigel, MD*, Madeline Carroll, MD², Christine Fanning, MD², Michael B. Steinberg, MD, MPH², \\ Amay Parikh, MD, MBA, MS ${ }^{3}$, Michael Usher, MD, $\mathrm{PhD}^{4}$
}

\begin{abstract}
'Division of Education and General Internal Medicine, Rutgers-Robert Wood Johnson Medical School, New Brunswick, New Jersey; ${ }^{2}$ Division of General Internal Medicine, Rutgers-Robert Wood Johnson Medical School, New Brunswick, New Jersey; ${ }^{3}$ Division of Critical Care and Nephrology, Rutgers-Robert Wood Johnson Medical School, New Brunswick, New Jersey; ${ }^{4}$ Division of General Internal Medicine, University of Minnesota Medical School, Minneapolis, Minnesota.
\end{abstract}

BACKGROUND: Interhospital transfer is an understudied area within transitions of care. The process by which hospitals accept and transfer patients is not well described. National trends and best practices are unclear.

OBJECTIVE: To describe the demographics of large transfer centers, to identify common handoff practices, and to describe challenges and notable innovations involving the interhospital transfer handoff process.

DESIGN AND PARTICIPANTS: A convenience sample of 32 tertiary care centers in the United States was studied. Respondents were typically transfer center directors surveyed by phone.

MAIN MEASURES: Data regarding transfer center demographics, handoff communication practices, electronic infrastructure, and data sharing were obtained.

RESULTS: The median number of patients transferred each month per receiving institution was 700 (range, 250-2500); on average, $28 \%$ of these patients were transferred to an intensive care unit. Transfer protocols and practices varied by institution. Transfer center coordinators typically had a medical background (78\%), and critical care-trained registered nurse was the most prevalent (38\%). Common practices included: mandatory recorded 3-way physician-tophysician conversation (84\%) and mandatory clinical status updates prior to patient arrival (81\%). However, the timeline of clinical status updates was variable. Less frequent transfer practices included: electronic medical record (EMR) cross-talk availability and utilization (23\%), real-time transfer center documentation accessibility in the EMR (32\%), and referring center clinical documentation available prior to transport (29\%). A number of innovative strategies to address challenges involving interhospital handoffs are reported.

CONCLUSIONS: Interhospital transfer practices vary widely amongst tertiary care centers. Practices that lead to improved patient handoffs and reduced medical errors need additional prospective evaluation. Journal of Hospital Medicine 2016;11:413-417. (c) 2016 Society of Hospital Medicine
Transitions of care are major sources of preventable medical errors. Incomplete or inaccurate communication during handoffs is the root cause of many adverse events. ${ }^{1}$ In a prospective study, adverse events were found to occur during interhospital transfer up to $30 \%$ of the time. ${ }^{2}$ Furthermore, patients subject to interhospital transfer have longer length of stay and higher inpatient mortality, even after adjusting for mortality risk predictors. ${ }^{3}$ Standardizing intrahospital handoff structures and communication practices has been shown to reduce medical errors. ${ }^{4-6}$ Interhospital transfer is an understudied area among the transitions of care literature. Little is known about institutional variations in

\footnotetext{
*Address for correspondence and reprint requests: Dana Herrigel, MD, Rutgers-Robert Wood Johnson Medical School, 1 Robert Wood Johnson Place, MEB Room 494, New Brunswick, NJ 08901; Telephone: 732-2356501; Fax: 732-235-7427; E-mail: dana.herrigel@rutgers.edu

Additional Supporting Information may be found in the online version of this article.

Received: October 28, 2015; Revised: January 15, 2016; Accepted: January 17, 2016

2016 Society of Hospital Medicine DOI 10.1002/jhm.2577

Published online in Wiley Online Library (Wileyonlinelibrary.com).
}

the process of information transfer and its association with patient outcomes. Although it is challenging to ascertain the total burden of transferred patients, it has been estimated that 1.6 million inpatients originated at another facility. ${ }^{7}$ Additionally, approximately $5.9 \%$ of admissions to a representative sample of US intensive care units (ICU) originated from other hospitals. ${ }^{8}$ Patients are transferred between hospitals for multiple reasons beyond medical necessity, for example, to adjust for patient preferences, bed availability, and hospital staffing patterns. This creates a setting in which complex and often critically ill patients are subject to variable and sometimes ambiguous handoff processes. ${ }^{9}$

This survey of 32 tertiary care centers in the United States was undertaken to identify common practices in communication and documentation during interhospital patient transfers. Additional goals were to understand the structure of the handoff process, the role of the transfer center, and how electronic medical records (EMR) and interhospital communication play a role in this care transition. Subsequently, common challenges in coordinating interhospital transfers were identified to provide a conceptual framework for process improvement. 


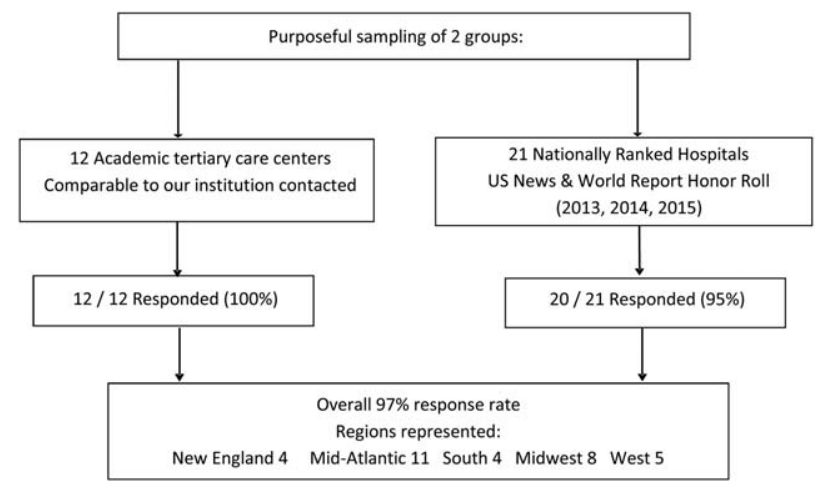

FIG. 1. Purposeful sampling conducted on 2 different groups of hospitals.

\section{METHODS}

\section{Survey Process}

The survey was initiated in September 2013 and concluded in September 2015, and was designed to quantify patient volume and identify common as well as unique practices to improve communication across the transfer process. The respondents were transfer center directors or managers, typically with a nursing background. Mass e-mail generated a very poor response rate and did not allow for discussion and clarification of responses. The strategy was then modified to contact individual institutions directly. The survey was performed via phone whenever possible. Figure 1 represents purposeful sampling conducted on 2 different groups of hospitals. These hospitals represent a convenience sample of institutions from a nationally ranked list of hospitals as well as others comparable to our own institutions. Hospitals were selected based on status as academic tertiary care centers with roughly similar bed sizes ( 600). Several were selected based on similar EMR capabilities. Geographic diversity was also taken into account. Thirty-two academic tertiary care centers were ultimately included in the survey. Data were entered into a survey form and deidentified. The Rutgers-Robert Wood Johnson Medical School Institutional Review Board approved this study.

\section{Survey Content}

Qualitative and quantitative data were collected by the study team. Data included number and origin of transfers (including those from inpatient facilities and emergency departments), staff characteristics, transfer process, documentation received prior to transfer, EMR access and type, outcomes, and clinical status tracking (see Supporting Figure 1 in the online version of this article for the complete survey tool).

\section{Measurement and Data Analysis}

Descriptive statistics are presented in unweighted fashion as a number and percentage for dichotomous variables, or a numeric range for ordinal variables. When a range was given by survey participants, the lower end of the range was used to calculate the population median. Several institutions surveyed were unable to provide specific numeric values, but instead cited how many requests for transfer they received either daily or monthly; these were omitted from the demographics analysis.

Respondents also provided a description of their overall triage and acceptance process for qualitative analysis. Unique strategies were identified by the study personnel at the time of each interview and amassed at the end of the interview period. These strategies were then discussed by the study team, and separated into categories that addressed the main challenges associated with interhospital transfers. Five general tenants of the transfer process were identified: acceptance and transport, need for clinical updates, provider handoffs and coordination of care, information availability, and feedback.

\section{RESULTS}

Based on a survey question asking respondents to estimate the total number of interhospital transfers received per month, the annual burden of patients transferred into these 32 hospitals represented approximately 247,000 patients yearly. The median number of patients transferred per month, based on a point estimate if given or the lower end of the range if a range was provided, was 700 (range, 250-2500). On average, $28 \%$ (range, $10 \%-50 \%$ ) were transferred directly to an ICU, representing approximately 69,000 critically ill patients. A majority of hospitals polled $(65 \%)$ received patients from more than 100 referring institutions, and a minority $(23 \%)$ identified EMR interoperability for more than a quarter of the sending facilities. The overall acceptance rate ranged from $50 \%$ to $95 \%$.

Table 1 represents common transition elements of participating institutions. Thirty-eight percent of hospitals utilize a critical care-trained registered nurse as the initial triage point of contact. The process and quality controls for coordinating transfers from outside hospitals were highly variable. Although clinical updates from acceptance to arrival were required in a majority of hospitals $(81 \%)$, the acceptable time interval was inconsistent, varying from 2 to 4 hours (13\%) to 24 hours $(38 \%)$. A mandatory 3-way recorded discussion (between transfer center staff, and referring and accepting physician) was nearly uniform. Objective clinical information to assist the handoff (ie, current labs, radiology images, history and physical, progress notes, or discharge summary) was available in only $29 \%$ of hospitals. Only $23 \%$ of hospitals also recorded a 3-way nursing handoff (bedside-to-bedside nursing report). A minority of hospitals utilized their principal EMR to document the transfer process and share incoming clinical information among providers $(32 \%)$. 


\begin{tabular}{|c|c|c|}
\hline Survey Question & Survey Response & $\mathrm{N}(\%)$ \\
\hline \multirow{3}{*}{$\begin{array}{l}\text { What is the training background of the staff member who takes the } \\
\text { initial call and triages patients in your transfer center? }\end{array}$} & Critical care experienced RN & $12 / 32(38 \%)$ \\
\hline & Other clinical background (EMT, RN) & 13/32 (41\%) \\
\hline & Nonclinical personnel & $7 / 32(22 \%)$ \\
\hline \multirow{2}{*}{$\begin{array}{l}\text { Prior to the patient's arrival, do you require any documentation } \\
\text { to be transmitted from the transferring institution? }\end{array}$} & Objective clinical data required & $9 / 32(28 \%)$ \\
\hline & Objective clinical data not required & $23 / 32(72 \%)$ \\
\hline \multirow[t]{2}{*}{ Is a 3-way recorded conversation facilitated by the transfer center required? } & Initial physician-to-physician acceptance discussion & $27 / 32(84 \%)$ \\
\hline & RN-to-RN report & $6 / 26(23 \%)$ \\
\hline \multirow[t]{5}{*}{ Are clinical status updates required? } & Updates required every 24 hours & $12 / 32(38 \%)$ \\
\hline & Updates required every 8-12 hours & $7 / 32(22 \%)$ \\
\hline & Updates required every 2-4 hours & $4 / 32(13 \%)$ \\
\hline & Updates required but timing not specified & $3 / 32(9 \%)$ \\
\hline & Clinical status updates not required & $6 / 32(19 \%)$ \\
\hline \multirow{2}{*}{$\begin{array}{l}\text { Is any clinical information obtained by the transfer center available to } \\
\text { the patient's providers in real time on your EMR system? }\end{array}$} & Yes & $10 / 31(32 \%)$ \\
\hline & № & $21 / 31(68 \%)$ \\
\hline \multirow[t]{2}{*}{ Do you track the outcomes of patients you accept from outside hospitals? } & Yes & $14 / 24(58 \%)$ \\
\hline & № & $10 / 24(42 \%)$ \\
\hline
\end{tabular}

NOTE: The denominator is the number of institutions who had available data and responded to that particular question. Abbreviations: EMR, electronic medical record; EMT, emergency medical technician; RN, registered nurse.

Descriptions of the transfer process were conceptually evaluated by the study team, then divided into 5 common themes: acceptance and transport, clinical updates, coordination of care, information availability, and quality improvement (Table 2). Institutions devised novel approaches including providing high bed priority to expedite transit, a dedicated "quarterback" physician to coordinate safe transfer and uninterrupted communication, electronic transfer notes to share communication with all providers, and a standardized system of feedback to referring hospitals. Several institutions relied on an "expect note," which could be a free-text document or a form docu- ment in the EMR. This preserves verbal handoff information that may otherwise be lost if the accepting physician at the time of transfer is not the physician receiving the handoff.

Quality improvement occurred via both internal and external feedback at several institutions. There were two notable mechanisms of internal feedback. Review of recorded physician verbal handoff by department chair occurred if an adverse event involved a transferred patient. An automatic internal review was triggered if a patient was upgraded to a higher level of care within 4 hours of arrival. These advanced mechanisms require vigilance and dedication

TABLE 2. Qualitative Survey Results: Challenges and Innovations

\begin{tabular}{|c|c|}
\hline Challenges & Innovative Practices \\
\hline \multirow[t]{4}{*}{ Expedited acceptance and transport } & Automatic acceptance for certain diagnoses (ie, neurosurgical indication for transfer) \\
\hline & Transferred patients prioritized for hospital beds over all patients except codes \\
\hline & Hospital controls transportation units, allowing for immediate dispatch and patient retrieval \\
\hline & Outsourcing of transfer center and interfacility transfer to third party \\
\hline \multirow[t]{4}{*}{ Timeliness of clinical updates } & Transfer center communicates with bedside RN for clinical updates at the time of transfer \\
\hline & Clinical status updates every 2-4 hours for critical patients \\
\hline & Daily reevaluation of clinical status \\
\hline & Accepting physician alerted of changes in clinical status \\
\hline \multirow[t]{3}{*}{ Handoff and coordination of care } & "Physician accept tool" in EMR \\
\hline & "Quarterback" physician who triages and accepts all patients during a given time period \\
\hline & $\begin{array}{l}\text { Critical patients are accepted into a "critical care resuscitation unit," an all-purpose intensive care unit } \\
\text { staffed by an intensivist who shares decision making with the referring provider and is involved in } \\
\text { all communications regarding the transferred patient }\end{array}$ \\
\hline \multirow{5}{*}{$\begin{array}{l}\text { Availability of protected clinical } \\
\text { information }\end{array}$} & Scribed physician handoff imported into EMR \\
\hline & "Expect note" in EMR: summary of clinical information documented by accepting physician \\
\hline & PACS radiology cloud networks for hospital systems or statewide \\
\hline & EMR interoperability: "Care Everywhere" module in Epic EMR \\
\hline & Health and information management department responsible for obtaining and scanning outside records into EMR \\
\hline \multirow[t]{5}{*}{ Feedback and quality improvement } & Automatic review if patient upgraded to ICU within 4 hours of arrival \\
\hline & Departmental chair review of physician verbal handoff if poor outcome or difficulty with transfer \\
\hline & Outcomes and quality of handoff reported back to referring hospital \\
\hline & Discharge summary sent to referring hospital \\
\hline & Referring hospital able to view patient's chart for 1 year \\
\hline
\end{tabular}


on the part of the transfer center and physicians involved in the transfer process. External feedback was provided to referring hospitals through both active and passive mechanisms. One advanced health system allowed referring providers to access the patient's inpatient medical record for 1 year and sent a discharge summary to all referring hospitals. Another hospital maintained a sophisticated "scorecard," with key measures shared with internal stakeholders and referring hospitals. Some of the metrics tracked included: denials due to insufficient bed capacity, change in bed status within 12 hours of transfer, and duration of stay in the postanesthesia care unit or emergency department awaiting an inpatient bed. This organization also performed site visits to referring hospitals, addressing handoff quality improvement.

\section{DISCUSSION}

Standardizing intrahospital handoffs has been shown to decrease preventable medical errors and reduce possible near-miss events. ${ }^{6,10}$ Interhospital care transitions are inherently more complex due to increased acuity and decreased continuity; yet, there is no universal standardization of these handovers. We found that practices vary widely among tertiary care centers, and the level of transfer center involvement in the verbal and written handoff is inconsistent.

Evidence-based frameworks to improve healthcare delivery, such as TeamSTEPPS (Team Strategies and Tools to Enhance Performance and Patient Safety), first require an organizational assessment to identify barriers to effective communication. ${ }^{11}$ Interhospital transfers offer multiple unique barriers to continuity: physical distance, uncertainty in timing, incongruent treatment goals, disparate information sources, and distractions. This study provides the first step in conceptualizing the unique aspects of interhospital transfers, as well as highlights strategies to improve care coordination (Table 2).

A tailored intervention needs not only to overcome the typical barriers to handoffs such as time constraints, information sharing, and ambiguity in provider roles, but also to overcome multiple systems barriers. Bed management systems add another timerelated variable due to fixed and frequently overburdened bed capacity. Prioritization of transfers depends upon an accurate clinical depiction of patient acuity as well as organizational strategies. For example, neurologic diagnoses are commonly a top priority and are triaged as such, sometimes instead of higher-acuity patients with other principal diagnoses. The complexity of this process may lead to delays in high-acuity transfers, and is contingent upon accurate and updated clinical information. Coordinating handovers amidst complex provider schedules is another systems barrier. The commonly adopted "7 on, 7 off" model for hospitalists, and shift work for intensivists, may increase the possibility that a transfer occurs across multiple provider changes. Patient follow-up and closed-loop feedback are important components of intrahospital handovers, but are much more challenging to implement for interhospital handovers with incongruent information systems and providers.

Programs to improve intrahospital handovers (eg, IPASS) emphasize creating an accurate clinical depiction of a patient using both verbal and written handoffs. ${ }^{12}$ This is arguably more difficult over the phone without a concurrent written handoff. Recording of 3-way physician and nurse handoffs is common, but reviews of recorded conversations are often unavailable or cumbersome in real time. EMR documentation of verbal information exchanged during the handoff is a possible solution. However, there may be legal implications for a transcribed verbal handoff. Furthermore, transfer centers often work with a software program separate from the principal EMR, and documentation in real time is challenging. EMR integration could help reinforce a patient-centered shared mental model by allowing visualization of lab trends, radiology, vitals, and other documentation during and after the verbal handoff.

Physician-driven checklist "accept tools" are another solution. Usually the responsibility of the accepting attending or fellow, this type of document is most useful as a modifiable document in the EMR. Accept tools, such as the one created by Malpass et al., have demonstrated successful shared decision making, and have resulted in fewer emergent procedures and fewer antibiotic changes on arrival. ${ }^{13}$ One of the challenges with this approach is the frequency of utilization. In the aforementioned study, the adoption rate of the accept tool was about $70 \%$ in a closed university medical ICU, where these types of interventions may be viewed favorably by providers instead of burdensome. ${ }^{13}$

The most consistent finding of this survey was the lack of common processes to improve outcomes. Simple interventions, such as regular clinical updates, documentation of the handoff process, and obtaining objective information early in the process, were inconsistently adopted. Outcomes tracking and feedback are necessary components of team-based quality improvement. Approximately half of the hospitals surveyed specifically tracked outcomes of transferred patients, and a minority had systems in place to provide feedback to referring centers.

Improving care delivery requires buy-in from all participants, necessitating engagement of referring hospitals. Interventions such as frequent status updates and providing early documentation have the potential to increase the burden on referring providers when feedback or incentives are not commonplace. Moreover, the referring provider has the option of transferring a patient to a hospital with reduced handoff requirements, creating a disincentive for quality improvement. Quality metrics that incorporate outcomes of 
transferred patients may be necessary to better align the goals of sending and receiving physicians.

This study was intended to be a qualitative investigation and has some limitations. Any verbal qualitative study has the possibility of misinterpretation of information given by transfer center personnel. A single investigator performed most of the discussions and was able to clarify when needed, providing a degree of consistency, but may also be a source of bias. Categorical answers and a team-based approach to conceptualizing responses likely minimized this potential bias.

We selected hospitals from the U.S. News and World Report Honor Roll plus additional hospitals chosen based on similarity to our home institutions. This may be a skewed sample and may not represent other major US hospitals and networks. However, we chose to interview large academic tertiary care centers, many accepting more than 1000 patients monthly, as these are likely to be the most proficient at performing transfers, and responses may be generalizable.

\section{CONCLUSIONS}

Standardization of information exchange during interhospital transfers does not currently exist. Practices vary widely amongst academic tertiary care centers. There is a paucity of data to support the association of specific processes with patient outcomes. Ultimately, a multicenter study examining the impact of improved information transfer on patient outcomes is warranted, utilizing tracking resources already in place. Optimizing and aligning practices between sending and receiving hospitals may improve interhospital handover efficiency and patient safety.

Disclosures: Dr. Usher is supported by a National Institutes of Health Clinical and Translational Science Award at the University of
Minnesota: UL1TR000114. Dr. Steinberg has received support from Arena Pharmaceuticals and Major League Baseball. Drs. Herrigel, Parikh, Fanning, and Carroll have no disclosures. A prior version of this article was presented as an abstract at the Society of General Internal Medicine Mid-Atlantic Regional Meeting in April 2014 in New York, New York.

\section{References}

1. Raduma-Tomas MA, Flin R, Yule S, Williams D. Doctors' handovers in hospitals: a literature review. BMJ Qual Saf. 2011;20(2):128-133.

2. Ligtenberg JJ, Arnold LG, Stienstra Y, et al. Quality of inter-hospital transport of critically ill patients: a prospective audit. Crit Care. 2005; 9(4):R446-R451.

3. Sokol-Hessner L, White AA, Davis KF, Herzig SJ, Hohmann SF. Interhospital transfer patients discharged by academic hospitalists and general internists: characteristics and outcomes [published online November 20, 2015]. J Hosp Med. doi: 10.1002/jhm.2515.

4. Nagpal K, Abboudi M, Fischler L, et al. Evaluation of postoperative handover using a tool to assess information transfer and teamwork. Ann Surg. 2011;253(4):831-837.

5. Starmer AJ, Sectish TC, Simon DW, et al. Rates of medical errors and preventable adverse events among hospitalized children following implementation of a resident handoff bundle. JAMA. 2013;310(21): 2262-2270.

6. Starmer AJ, Spector ND, Srivastava R, et al. Changes in medical errors after implementation of a handoff program. N Engl J Med. 2014; 371(19):1803-1812.

7. HCUP National Inpatient Sample (NIS). Healthcare Cost and Utilization Project (HCUP). 2012. Agency for Healthcare Research and Quality, Rockville, MD. Available at: www.hcup-us.ahrq.gov/nisoverview.jsp. Accessed 26 May 2015.

8. Wagner J, Gabler NB, Ratcliffe SJ, Brown SE, Strom BL, Halpern SD. Outcomes among patients discharged from busy intensive care units. Ann Intern Med. 2013;159(7):447-455.

9. Wagner J, Iwashyna TJ, Kahn JM. Reasons underlying inter-hospital transfers to an academic medical intensive care unit. J Crit Care. 2013;28(2):202-208

10. Payne CE, Stein JM, Leong T, Dressler DD. Avoiding handover fumbles: a controlled trial of a structured handover tool versus traditional handover methods. BMJ Qual Saf. 2012;21(11):925-932.

11. Keebler JR, Dietz AS, Lazzara EH, et al. Validation of a teamwork perceptions measure to increase patient safety. BMJ Qual Saf. 2014; 23(9):718-726.

12. Starmer AJ, O'Toole JK, Rosenbluth G, et al. Development, implementation, and dissemination of the I-PASS handoff curriculum: a multisite educational intervention to improve patient handoffs. Acad Med. 2014;89(6):876-884.

13. Malpass HC, Enfield KB, Keim-Malpass J, Verghese GM. The interhospital medical intensive care unit transfer instrument facilitates early implementation of critical therapies and is associated with fewer emergent procedures upon arrival. J Intensive Care Med. 2015;30(6): $351-357$. 